\section{WHITENING POWER}

Arm \& Hammer toothpastes are low abrasive and contain baking soda which is clinically proven to significantly enhance the plaque removal efficiency of toothbrushing compared to products without baking soda.

Arm \& Hammer Advance White deep cleans all areas of the teeth and banishes surface stains for brighter, whiter teeth. It is a low abrasion toothpaste due to the natural ingredient, baking soda, which is clinically recognised to gently maintain tooth enamel.

Used twice a day for 14 days, Advance White toothpaste lightens the teeth up to three shades. Plus, it has Micropolisher Technology for a shiny, healthy smile.

For more information about the carefully formulated Arm \&
Hammer toothpaste range, visit http:// www.armandhammer.co.uk/ or email: ukenquiries@churchdwight.com.

Arm \& Hammer oral healthcare products are available at Boots, Superdrug, Sainsbury's, Tesco, Asda and Morrisons throughout the UK.

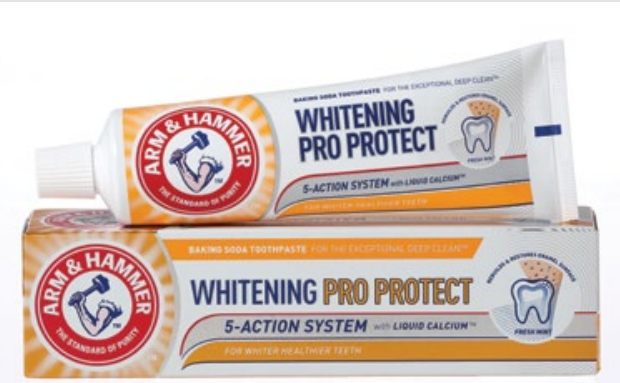

\title{
A COMPLETE DISINFECTION RANGE FOR DENTISTRY
}

EndoSan produce highly effective, broad spectrum hydrogen peroxide based disinfectants that form no harmful byproducts or residuals - degrading into water and oxygen, whilst offering unrivalled stability. It is chlorine and alcohol free, with no corrosive effects. EndoSan is a safe biocide that is truly biodegradable. It can be used for constant or shock dosing in dental unit water lines, fogging, surface disinfection, manual dosing, and hand sanitisation.

Endosan 3 is a surface disinfectant that is completely odourless, tasteless, and colourless making it the perfect application to wipe down cabinets, chairs, and all other hard surfaces within the dental practice.

Endosan 3 comes in a $500 \mathrm{ml}$ bottle with two

spray settings which is safe and easy for the user to apply.

Endosan 007 is a dental unit water line disinfectant that can be dispensed straight into the chairside bottle with no need to mix any potentially harmful chemicals. With just three uses of the hand pump (dosing 40 $\mathrm{ml}$ ) the 1 litre chair side bottle will be ready for use by topping up with either tap water or purified water. Endosan 007 can also be used to sanitise RO systems by clearing out all biofilm that may have built up, which will help prolong the life of future filters.

For more Endosan products such as Hand Sanitisers or to purchase the Endosan range call 01253 736355, email account@ dentaldecontamination.co.uk or visit www. dentaldecontamination.co.uk.

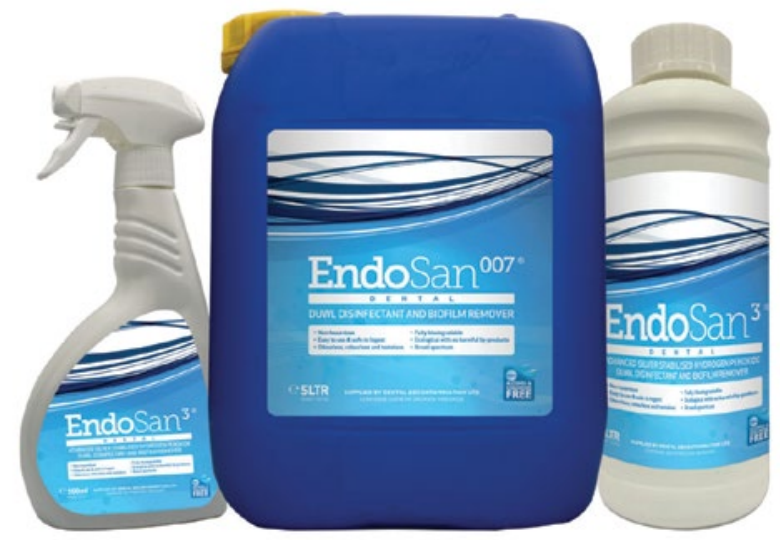

EYE PROTECTION THAT WON'T TICKLE YOUR EYELASHES

Protect yourself and your staff from aerosols and infectious respiratory droplets making contact with the conjunctiva.

Single use and ideal for infection control, Foamies form a seal on the forehead, nose and cheeks that does not allow infectious airborne material to enter the crevices typically found around traditional eyewear.

Extremely comfortable to wear longterm, Foamies are available in three sizes and with clear or tint options. They have no sharp edges or pressure points so do not cut into, hurt or irritate the nose, cheek or ears. Also, Foamies do not tickle the eyelashes as they stand away $12 \mathrm{~mm}$ due to the foam periphery. They will not fall off if you bend over.

All Foamies sizes are available in seethrough tint or clear: green (small) fits most children' blue (medium) fits most women; and yellow (large) fits most men.

Foamies and other oral care products are available through www.piksters.co.uk.

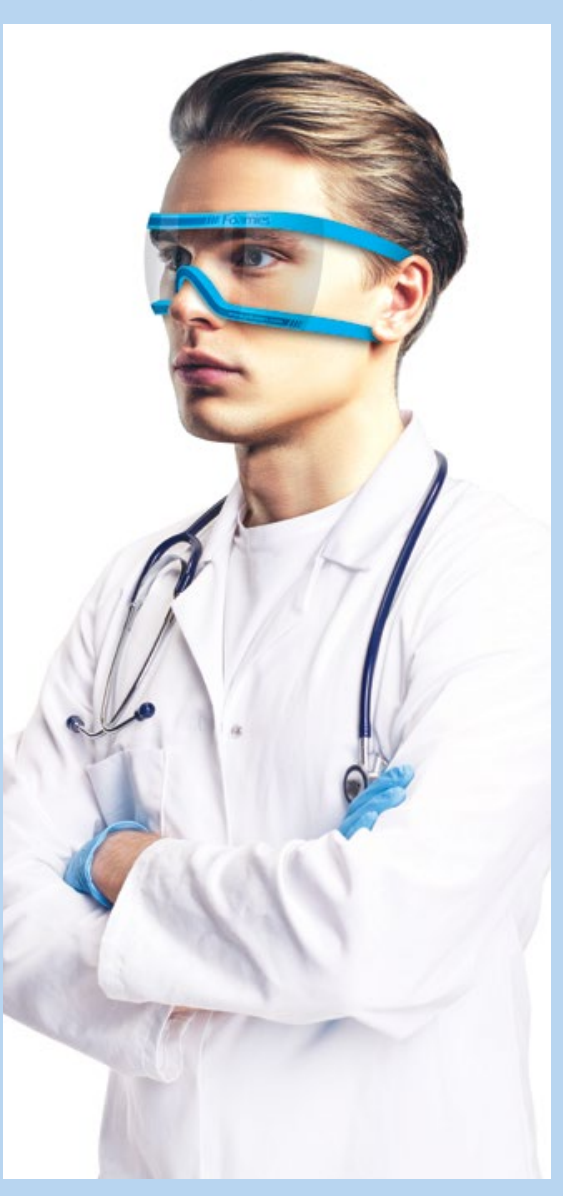

